\title{
LA ECONOMÍA LLEGÓ A LA ATMÓSFERA: EL RETO DE LA LEY MARCO DE CAMBIO CLIMÁTICO PARA LA ADAPTACIÓN COMO POLÍTICA PÚBLICA
}

ECONOMY HAS REACHED THE ATMOSPHERE: THE CHALLENGE OF THE FRAMEWORK CLIMATE CHANGE LAW FOR ITS ADAPTATION AS A PUBLIC POLICY

\author{
Gabriela Salvador Cárdenas* \\ Pontificia Universidad Católica del Perú
}

\begin{abstract}
Climate change is one of the main issues we face not only as a country but on a global scale, and it has challenged several countries to achieve a shifiting in state policies, forelooking the climate component's transversality.

Therefore, this article aims to argue the importance of a public policy oriented to climate change adaptation and the influence of the Framework Climate Change Law for the enforzability of decision making for the execution of such policy. Thus, since it is a challenge -as a country- to understand the need of adaptation policies, which are different from mitigation policies, towards the insurance of environmental sustainability for future generations. The climate change adaptation requires a transversal normative reformulation that allows the natural resources guard and the coexistence between environment and citizenship.
\end{abstract}

KEY WORDS: Climate change; adaptation; Paris Agreement; economy.
El cambio climático es uno de los principales problemas que afrontamos no sólo como país, sino a nivel mundial y que ha puesto en jaque a los diferentes estados a fin de lograr un viraje en las políticas estatales, con miras a la transversalidad del componente climático.

Es por ello que el objetivo del presente artículo es argumentar la importancia de una política pública orientada a la adaptación ante el cambio climático y, la influencia de la Ley Marco de Cambio Climático, para la obligatoriedad de la toma de decisión a fin de ejecutar dicha política. Ello, en tanto nos cuesta-como país- entender la necesidad de políticas de adaptación, distintas a la mitigación, con miras al aseguramiento de la sostenibilidad ambiental para las generaciones futuras. Así, la adaptación al cambio climático requiere una reformulación normativa transversal, que permita el resguardo de recursos naturales y la convivencia entre medio ambiente y ciudadanía.

Palabras clave: Cambio climático; adaptación; Acuerdo de París; economía.

\footnotetext{
Abogada. Magíster en Gobierno y Políticas Públicas de la Escuela de Gobierno y Políticas Públicas de la Pontificia Universidad Católica del Perú (PUCP). Asesora Parlamentaria. Adjunta del curso de Derecho de Pueblos Indígenas de la Facultad de Derecho de la PUCP. Contacto: gabrielasalvadorcardenas@gmail.com.
}

Nota del editor: El presente artículo fue recibido por el Consejo Editorial de THËMIS-Revista de Derecho el 31 de octubre de 2018, y aceptado por el mismo el 9 de enero de 2019. 


\section{INTRODUCCIÓN}

La problemática del cambio climático viene siendo abordada desde diferentes espacios, con el propósito de consolidarlo como un tema de agenda pública mundial. Esto último, con la finalidad de que los Estados adopten medidas para hacer frente a la problemática climática que viene generando impactos adversos al medioambiente y a la población en general.

En ese contexto, es necesario recordar que la Primera Conferencia de las Naciones Unidas que abordó temas ambientales se realizó en el año 1972 en la ciudad de Estocolmo y fue la entrada a la Primera Conferencia Mundial sobre el Clima en Ginebra, celebrada en 1979. En dicha conferencia, se habló por primera vez del cambio climático, por lo que se firmaron los primeros compromisos para hacer frente a este problema. Lo interesante de este hecho fue que los compromisos asumidos se hicieron bajo el precepto de que dichos cambios eran producto de la intervención del hombre. Estos eventos son dos puntos clave en la historia ambiental.

De tal manera que lo que vino después fue la intención de hacer frente al cambio climático de mano de la creación del Grupo Intergubernamental de Expertos sobre el Cambio Climático, la Organización Meteorológica Mundial y el Programa de las Naciones Unidas para el Medio Ambiente (en adelante, "PNUMA")

Las conferencias de las Naciones Unidas han formado la ruta a seguir en materia climática, cuyo punto cumbre fue la Conferencia de las Naciones Unidas sobre el Medio Ambiente y el Desarrollo, llevada a cabo en la ciudad de Río de Janeiro en 1992. Dicho evento permitió la adopción de la Agenda 21, la cual-entre otros temas-planteó un enfoque de desarrollo sostenible con la finalidad de luchar contra el cambio climático, resguardo de la biodiversidad y la eliminación de emisión de sustancias tóxicas. La adaptación aún no era la vía.

En esta línea de reuniones e intereses conjuntos se instalan las Ilamadas Conferencias de las Partes (en adelante, "COP"), la primera de las cuales fue realizada en Berlín en 1995 y que tuvo como participantes a las naciones que firmaron la Convención Marco de las Naciones Unidas para el Cambio Climático (en adelante, "CMNUCC").

Ahora bien, el Protocolo de Kioto, suscrito en el año 1997, fue el primer documento oficial en mostrar el cambio climático no solo desde la necesidad de la conservación y mitigación, sino también en términos de adaptación (BBC Mundo, 2005). No obstante, el daño al ambiente estaba hecho. Asimismo, el Protocolo de Kioto formó parte de la CMNUCC y significó el principal compromiso asumido ante el cambio climático, ya que planteó la reducción de gases de efecto invernadero. Así, los países que en ese momento eran denominados "industrializados" debían comprometerse, de ser el caso junto con los privados, a la reducción de sustancias tóxicas y buscar medidas de adaptación.

Conferencias como la de Bali en el año 2007 prepararon el camino para el diseño de los compromisos en mitigación, tecnología, financiamiento climático y adaptación, los cuales sirvieron para la reunión preparatoria de las decisiones en la Conferencia de Copenhague del año 2009. Cabe señalar que el Perú no estuvo exento de ser sede de uno de eventos. Es así, en el año 2014 se realizó la COP20 en Lima, la cual permitió un despliegue mediático de la responsabilidad ante los efectos climáticos y, además sirvió para sentar las bases de la siguiente conferencia de las partes que, posteriormente, se convertiría en el Acuerdo de París.

Si bien el Protocolo de Kioto fue el primer documento oficial en presentar el tema del cambio climático, el Acuerdo de París sí lo fue en términos de compromisos específicos, en los cuales la orientación de la economía debía dar un viraje, a fin de poder asumir dichos compromisos. Cabe indicar que el Perú es uno de los países firmantes de dicho Acuerdo, por lo que, incorporar el componente de adaptación a las políticas públicas -desde normas o planes de acción- es una obligatoriedad.

Sin embargo, en el Perú, asumir la vulnerabilidad y el riesgo ante un fenómeno climático es también un factor paradigmático. Ello, en tanto nuestra institucionalidad ambiental no está fortalecida ni viene desarrollándose de manera transversal. Mas aun, solo el Ministerio de Economía y Finanzas (en adelante, "MEF") incorporó en el 2013 -de manera superficial- el componente climático a la Gestión de Riesgos. Asimismo, el debate en el Congreso de la República durante el pleno del 15 de marzo del año pasado (Congreso de la República del Perú, 2018) develó la falta de información sobre el tema, en nuestros legisladores; toda vez que, en el desarrollo del debate, no se entendía a qué debíamos adaptarnos ni cómo debía ser entendida dicha adaptación.

El fenómeno del Niño, de tipo hidrometeorológico, que en marzo de 2017 azotó diferentes regiones del país, entre las cuales se encontraba Lima, permitió un antes y un después en materia climática. Los efectos devastadores de dicho fenómeno fueron relacionados de manera integral con el cambio climático. Así, la adaptación no podía seguir siendo 
una opción. La adaptación se convertía en una obligación, la cual es difícil de atender aun cuando -como país- presentamos un alto riesgo de vulnerabilidad, como ha sido reconocido en la CMNUCC.

Quizás ese escenario permitió que el debate en la agenda pública sobre el cambio climático nos propusiera nuevos retos como país. En ese sentido, el derecho al medio ambiente debe entenderse como un derecho fundamental y apuntar a una regulación que cumpla con la función social del Derecho y que, a la vez, sea acorde a las necesidades públicas.

Por ello, la Ley 30754 (en adelante, "Ley Marco de Cambio Climático") permitirá - una vez reglamentada- establecer criterios y puntos fijos para la adaptación ante este fenómeno. Tarea pendiente que deberá ser asumida por actores estatales y no estatales, en el marco de una defensa de derechos fundamentales y, de ser el caso, colectivos.

Sin embargo, el reto fundamental está en introducir en la política pública, desde desarrollos normativos o restructuración de las normas ya existentes, el componente de adaptación con relación al cambio climático. He ahí el aporte de la investigación, en entender que -como país- somos vulnerables al cambio climático y debemos tener herramientas legales que permitan la adaptación a dicho fenómeno. Asimismo, es de recordar que, entre las cinco categorías de las necesidades a la adaptación, se encuentra la institucional, en la cual la coordinación intergubernamental es la base para lograr la misma (Magrin, 2015).

\section{DE LA ADAPTABILIDAD ANTE EL CAMBIO CLIMÁTICO: EL ACUERDO DE PARÍS Y LA RE- FORMULACIÓN DE NUESTRA POLIITICA PÚ- BLICA PARA SUBSISTIR}

Una de las consecuencias del cambio climático es haber puesto en relieve la necesidad de la adaptación como un mecanismo para hacer frente a sus efectos -intentando reducir el impacto- y reorientar la economía a fin de que esta contemple entre sus lineamientos los componentes ambiental y climático.

En ese sentido, el Acuerdo de París cumple un rol fundamental en el camino trazado por los países miembros de la Conferencia de las Partes, en tanto resaltó la relación entre factores sociales y económicos que, aun cuando estuvieron siempre presentes, no estuvieron equilibrados en las políticas públicas. Esto es, se priorizó el crecimiento económico, sin contar con una regulación que permitiera la relación entre ambiente y economía responsable, hacia la sostenibilidad ambiental.
Así, señalaremos algunos aspectos indicados por el Acuerdo de París (Naciones Unidas, 2015) que sirvieron como base para la asunción de compromisos. Por un lado, las partes reconocieron que debían adoptar medidas específicas para hacer frente al problema del cambio climático. Medidas que debían tener -entre otros- un enfoque de derechos humanos, de género, interculturalidad y ser orientadas a la atención de poblaciones que se encuentren en mayor estado de vulnerabilidad.

Por otro lado, el Acuerdo de París, fue crítico ante el modelo de desarrollo que venimos implementando como país. Modelo que, de acuerdo con Iguiñiz, en tanto es planificado desde la visión de la productividad, encuentra una barrera con el desarrollo sostenible, toda vez que los recursos y ecosistemas tienen un límite; situación que, sin duda alguna, el cambio climático ha permitido develar (1998). Esta tesis, en el 2004, también es sostenida por Gudynas, en tanto indica que la devastación ambiental, por lo menos en Sudamérica, se explica en función a la presencia de una política de aprovechamiento de recursos que viene siendo inadecuada (2004).

En ese sentido, el documento destaca en tanto visibiliza un debate antiguo. La relación "intrínseca que existe entre las medidas, las respuestas y las repercusiones generadas por el cambio climático y el acceso equitativo al desarrollo sostenible y la erradicación de la pobreza" (UNFCC, 2015). A ello, suma el Acuerdo en mención el que las partes deben tener en consideración la seguridad alimentaria -la cual debe ser preservada- ante la vulnerabilidad de la producción alimenticia y, así afrontar la hambruna. Situación que como país se ha venido viviendo en los últimos años ante el estrés hídrico en zonas que antes fueron agrícolas, como Moquegua, Cajamarca o Arequipa.

Lo señalado en los párrafos precedentes podría ser entendido desde el concepto de justicia climática. Esta ve al cambio climático desde la perspectiva de los derechos humanos (Mendoza, 2009). Así, la justicia climática y la justicia ambiental son dos conceptos que permiten entender las aristas éticas y políticas que existen en torno a los problemas ambientales. Ello, es explicado además por Lanegra Quispe como la justificación de la presencia estatal en materia de regulación ambiental, ante las fallas del mercado, para que este sea eficiente y, a la vez, se logren reducir los niveles de pobreza vinculados a injusticias e inequidades, que tienen como factor secundario la materia ambiental (2008).

Ahora bien, volviendo a lo señalado por el Acuerdo de París como base para los compromisos, nos detendremos en la relación entre acciones frente 
al cambio climático y la erradicación de la pobreza. La pregunta entonces es ¿por qué si estamos frente a un tema ambiental, hablamos de términos económicos y de pobreza? La respuesta no es tan difícil de explicar, pero sí de entender desde los términos de desarrollo que imperan en diferentes países, entre los cuales se encuentra el Perú. Así, la respuesta evoca a paradigmas que se encuentran arraigados y con los que, desde la función social del derecho, debemos contribuir para su erradicación o transformación.

Porque el cambio climático es una consecuencia también de un modelo de desarrollo global que contribuye a la generación de gases de efecto invernadero (CEPAL, 2012). En términos paradójicos, la economía también llegó a la atmósfera. Ahora bien, esta idea de causa-efecto nos lleva a visibilizar otros conceptos, que serán útiles para explicar la adaptación. Esto es, la vulnerabilidad social y la inequidad que sostienen la violencia estructural en países como el Perú y que ponen en mayor riesgo a poblaciones que no cuentan con recursos ni servi- cios básicos que permitan -mínimamente- resistir los impactos ambientales. Mas aun cuando estas poblaciones tienen concepciones culturales diferentes que no son consideradas en el modelo de desarrollo que se impone y el acceso a los recursos naturales es priorizado para actividades económicas y no desde una perspectiva de sostenibilidad o aseguramiento de generaciones futuras.

Es por ello que, en el caso peruano, en el cual la diversidad cultural es tan amplia, las respuestas al frente climático no pueden dejar de verse desde la perspectiva intercultural. Sobre este punto, retomaremos en el siguiente acápite.

Ahora bien, de acuerdo con la CMNUCC, son nueve las características que hacen que un país sea vulnerable. En el gráfico adjunto, presentamos las recogidas en el artículo 8 de la CMNUCC:

De las nueve características señaladas, el Perú presenta siete de ellas, lo cual nos ubica como un país de alto riesgo ante los impactos del cambio

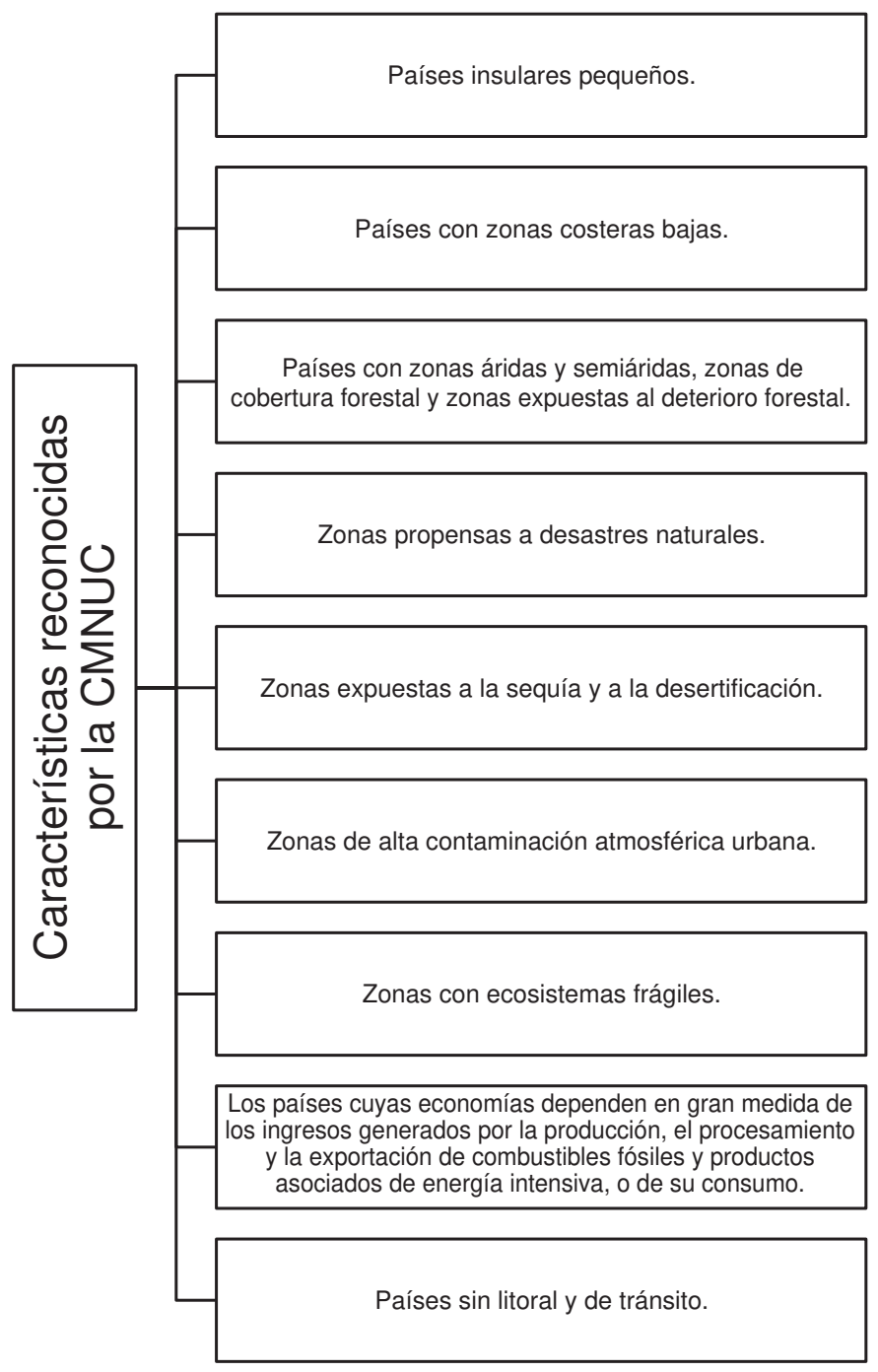


climático y, por ende, con mayor responsabilidad como Estado para hacer frente a los mismos. Es por esta razón que, desde el Estado, debemos aplicar herramientas legales en las políticas públicas que nos permitan hacer frente al cambio climático, pero no solo desde la mitigación, sino con miras a la adaptación, es decir, a la aseguración de espacios para generaciones futuras.

Ahora, la adaptación es uno de los quehaceres ante el cambio climático. Esta medida busca hacer una reingeniería de lo actuado. Así, el marco jurídico, medios de vida, diseño de infraestructura, pasan por una decisión política orientada a responder a los efectos e impactos del cambio climático; los mismos que cuentan ya con una proyección dada por expertos en la materia. Eventos que pueden acarrear sequías, desbordes, entre otros.

Así, la adaptación fue definida por el Grupo Intergubernamental de Expertos sobre el Cambio Climático como el mecanismo mediante el cual un país hace diferentes ajustes de orden económico, social, estructural, con miras a hacer menos nocivo el daño ante los efectos climáticos y, en algunos casos, ver oportunidades de mejora ante la crisis climática. Uno de estos cambios, por ejemplo, se daría en materia energética, en la cual nuestra regulación aún es incipiente. Así, pese a contar con una norma que promueve las energías renovables, la subasta con relación a estas aún no ha sido implementada. Esto demuestra que la norma, por sí sola, no genera el cambio. En materia energética, el desafío es grande, histórico y paradigmático. La promoción está orientada al uso de contaminantes para la producción de energía, incluso, en uso doméstico, lo cual conlleva a sistemas ineficientes que tienen impactos directos en la salud e inequidad, pero, sobre todo, en la emisión de gases de efectos invernaderos, como el carbono y la deforestación (Mulugetta, Been Haga y Kammen, 2019).

Finalmente, sobre este punto, Watt es clara en señalar qué hacer frente al cambio climático: parte de cambios estructurales. Así, señala que los paradigmas pueden, como la cultura, evolucionar; pero ello parte de reconocer, como sociedad y estado, compromisos consecuentes para frenar dicho fenómeno. Esto es, aprender a adaptarnos (2016).

\section{A. La adaptación no es igual para todos: la difí- cil ruta de los países en desarrollo}

Si bien la adaptación es un tema que abarca a todos los países, en algunos casos, su logro demanda mayores esfuerzos. Así, un país que goza de estabilidad económica, política y que además presente menor grado de vulnerabilidad ante el cambio climático tendrá mejores herramientas para adaptarse al mismo. Zwarteveen suma a ello el que los países deberán negociar "costos y responsabilidades" por el cambio climático; etapa en la cual aquellos países que son más vulnerables ante dichos efectos son también los que cuentan con menos posibilidades de negociar (2013).

Ese beneficio no sucede con los denominados países en desarrollo o que se encuentran en un nivel de vulnerabilidad mayor. En ese sentido, en casos como el peruano, por ejemplo, cuyo modelo económico depende del uso de recursos naturales, se deberá invertir en mayor medida en herramientas de adaptación. Esto requiere principalmente de una decisión política que permita el viraje a un modelo de desarrollo que tenga un enfoque de sostenibilidad ambiental y que permita entender la justicia ambiental y climática como una vía para el cambio, pero desde el enfoque de adaptación.

En la línea de lo señalado en el párrafo precedente, la adaptación y cambio climático es también una forma de hablar de justicia social, por lo que se debe generar un cambio en la adopción de soluciones públicas, cambio que debe tener un enfoque de derechos (Zwarteveen, 2013); el mismo que a su vez debe considerar componentes ambientales y culturales. Así, en un espacio en el cual el clima tiene una alta variación, como la zona andina, las herramientas de adaptación deberán considerar la seguridad alimentaria y aseguramiento hídrico, por lo que entender la importancia de la preservación de las cuencas y sus cabeceras es imperante. Más aún, si vamos hacia un camino de estrés hídrico. Lo anterior, debe ser entendido desde la realidad, ante casos de friaje o sequías en la zona andina, que además sufre los impactos de actividades económicas, en las cabeceras de cuenca y que vuelven a los ecosistemas menos resilientes.

Hasta este punto, es claro que la adaptación no es igual para todos los países, incluso en temas de costo. Por ello, el Grupo Intergubernamental de Expertos sobre el Cambio Climático sostiene la necesidad de la financiación para la adaptación, la cual no debe ser entendida solo como el auxilio de un país a otro ante emergencias ambientales, sino más bien como la búsqueda de la sostenibilidad ambiental. Así, existen diferentes fondos que tienen el fin propuesto: (i) el Fondo Fiduciario para el Medio Ambiente Mundial (en adelante, "FMAM"), (ii) el Fondo Especial sobre el Cambio Climático, (iii) el Fondo para los Países Menos Adelantados, (iv) el Fondo de Adaptación ${ }^{1}$ y (v) el Fondo Verde para el Clima.

Los fondos (ii), (iii) y (iv) fueron creados en el marco del Protocolo de Kioto. 
Ahora bien, como ya hemos señalado anteriormente, el Acuerdo de París es probablemente el documento oficial más importante en materia de cambio climático, en tanto recoge compromisos de diferentes países. Uno de ellos, de manera implícita, reconoce la diferencia entre países para hacer frente a los impactos de cambio climático y asumir medidas de adaptación. Así, el Acuerdo de París busca asegurar la disponibilidad de financiamiento con fines climáticos mediante mayores "flujos financieros" que permitan la resiliencia ante los efectos del clima y, además, una reducción en la emisión de gases con efecto invernadero. Cabe indicar que el Acuerdo de París, con relación al Fondo Verde, hizo un pedido especial con la finalidad de que se acelere el apoyo financiero a países que estaban en vías de desarrollo.

Adicionalmente, el Acuerdo de París, en materia de financiamiento para hechos tales como la adaptación, planteó el aumento del mismo para el período del 2020 hasta el 2025, con la finalidad de brindar apoyo a los países en desarrollo. Sin embargo, debemos señalar que el financiamiento, si bien ayuda, no es el único paso. Este debe ser utilizado de manera adecuada, lo cual, en casos como el peruano, no podrá lograrse si no se cuenta con un enfoque transversal del componente climático y una institucionalidad ambiental sólida.

\section{B. Tipos de adaptación}

La adaptación ante el cambio climático puede ser de diferente tipo. Actualmente, a nivel teórico, se reconocen seis formas de adaptación (ver gráfico adjunto).

Sobre el particular, el tipo de adaptación más común es la reactiva y autónoma. Sin embargo, documentos como el Acuerdo de París han permitido ampliar la mirada a la prevención para que, desde el actor estatal, se busque una política sostenida de adaptación. Algo que, como país, aún estamos lejos de aplicar, más aún cuando tenemos un sector-Ministerio del Ambiente- con debilidad institucional, tal como se ve reflejada en su presupuesto.

\section{Reactiva}

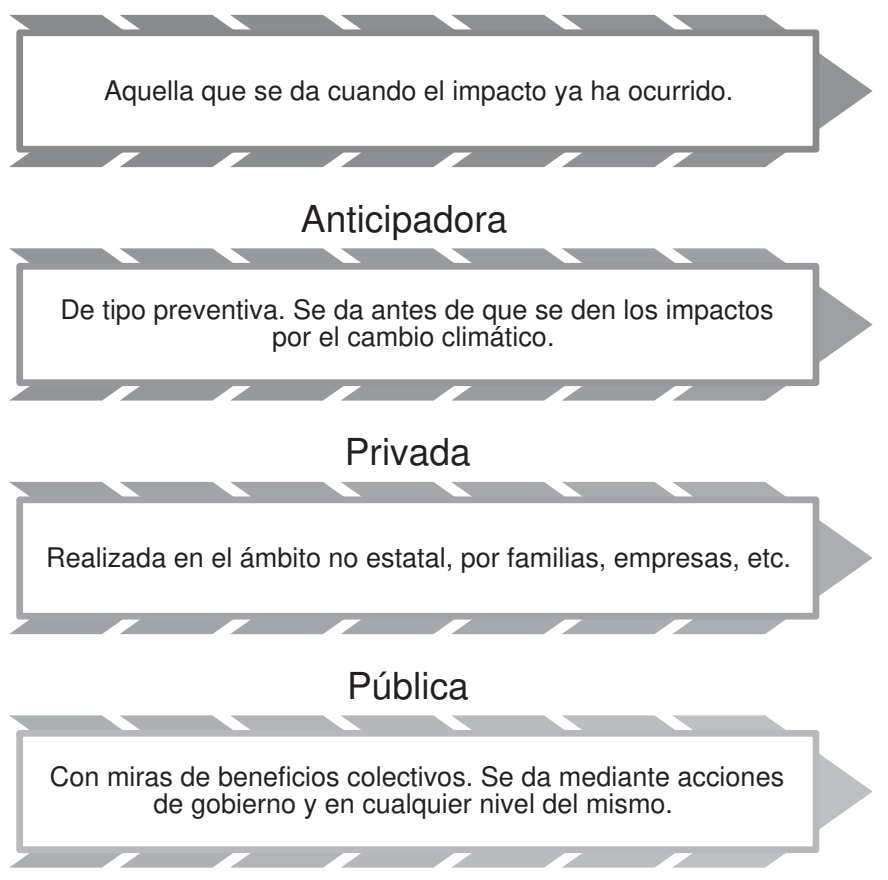

\section{Autónoma}

Es espontánea y se da ante cambios que pueden ser a nivel de
mercado o de tipo ecológico, natural; pero también ante compromisos internacionales.

\section{Planificada}

Es una decisión política. Se orienta a la formulación de planes nacionales o políticas públicas que parten de la idea de un cambio para hacer frente a los impactos climáticos. 
En esa línea, las Contribuciones Nacionales Determinadas (en adelante, "iNDC", por sus siglas en inglés) son un mecanismo para lograr la adaptación preventiva, planificada y pública, pero también se permite posicionar al actor no estatal en los compromisos. Así, la adaptación no es solo una tarea estatal. Si bien el país es quien debe impulsarla, el actor no estatal es quien debe también asumir tanto responsabilidad, como acciones concretas para hacer frente al cambio climático. En ese sentido, México es uno de los países que a la fecha ha presentado contribuciones que abarcan planes de adaptación.

\section{LA LEY MARCO DE CAMBIO CLIMÁTICO: EL RETO ESTÁ EN LA ADAPTACIÓN}

A modo de recuento, se ha señalado que el Acuerdo de París cobra especial importancia en tanto es el primer documento oficial en el cual los países miembros de la Conferencia de las Partes asumen compromisos para hacer frente a los impactos del cambio climático. Compromisos políticos y económicos que involucran a todos los niveles de gobierno y que además pone en escena a los actores no estatales.

Ahora bien, el Perú es uno de los pocos países en América Latina que cuenta con una norma interna específica para realizar acciones con relación al cambio climático. Norma que guarda coherencia con lo señalado en el Acuerdo de París y que orienta el camino a una política pública transversal climática, pero que a la fecha no cuenta con reglamento que permita su viabilidad. Cabe indicar que dicha norma fue el resultado de un esfuerzo ${ }^{2}$ de la Comisión de Pueblos Andinos, Amazónicos, Afroperuanos, Ambiente y Ecología (en adelante, "CPAAAAE") del Congreso de la República, durante la gestión 2017-201833, por lograr un consenso en los diferentes proyectos de ley que se presentaron con relación a materia climática, entre los que se encontraba el del propio MINAM. La Ley Marco de Cambio Climático fue promulgada el 17 de abril de 2018.

Sin embargo, tal como puede verse en los debates del dictamen de la norma, en el pleno del Congreso realizado el 11 de enero de 2018 la mayor resistencia fue en torno a la adaptación, la cual tuvo que ser explicada, en más de una oportunidad, técnicamente. Pese a ello, si bien se aprueba la norma, el concepto de adaptación es entendido solo para zona amazónica; sin embargo, el reto está en que la adaptación sea vista como un mecanismo de aseguramiento de nuestra propia existencia, con un enfoque intergeneracional.

Ahora bien, este hecho no es ajeno. Entender la adaptación como una realidad es -como lo hemos señalado anteriormente- algo que no termina por aceptarse, en tanto es paradigmático. El daño ya está hecho y lo que nos queda es mitigar, conservar y adaptarnos. Así, incluso en el marco de los acuerdos internacionales, los compromisos estatales aún no logran hacerse efectivos, pese a que los efectos del cambio climático sí lo son y tienen tendencia a ir magnificándose. Es por ello que la mitigación no es una herramienta altamente efectiva en este punto de la historia en materia de reducción de gases de efecto invernadero, sino más bien, la adaptación (Stratus Colsulting Inc., 2004). En ese sentido, la Ley Marco de Cambio Climático señala desde su objeto que permitirá establecer -entre otros- principios y enfoques para articular, diseñar y ejecutar las políticas públicas de las medidas de adaptación. La norma en mención presenta a la adaptación desde el enfoque social, político y económico. Este último incluso está presente en el principio de rendición de cuentas, en tanto la adaptación requiere de manejo de recursos.

Por otro lado, destaca el principio de gobernanza climática recogido en la Ley Marco de Cambio Climático. El Acuerdo de París indica que la adaptación es una tarea de todos, lo cual involucra a todos los niveles de gobierno. Por ende, el principio en mención se da en concordancia con dicho Acuerdo y plantea la participación efectiva de todos los actores públicos en la toma de decisiones. Asimismo, esta tarea involucra a los actores privados, a fin de lograr consensos. Cabe indicar que, si bien el principio de gobernanza de la Ley Marco de Cambio Climático abarca a actores estatales y no estatales, las metas y objetivos se dan en función a responsabilidades definidas.

Otro principio importante de la ley en mención es el de prevención. Si bien la prevención es un concepto distinto al de la adaptación, la norma presenta una relación importante entre ambos. Así, la norma traza la ruta de lo que serán las acciones de prevención, pero también identifica el daño ambiental, el cual es una realidad que vivimos día a día. Plantea que, ante aquellos casos en los que no se pueda revertir o eliminar las causas de generación de daño, deberán adoptarse -entre otrasmedidas de adaptación. Ahora bien, lo interesante

2 Durante la sesión ordinaria del 28 de noviembre de 2017 de la CPAAAAE se aprobó el dictamen de la Ley Marco de Cambio Climático.

3 La gestión de la CPAAAAE en la cual se aprobó el dictamen de la Ley Marco de Cambio Climático estuvo a cargo del congresista Marco Arana Zegarra. 
de esta propuesta es que esas medidas deberán tener un enfoque de derechos fundamentales, toda vez que tienen como base el resguardo de vida, salud y medioambiente. Así, la norma plantea también la ruta a la construcción de un discurso: el derecho a un ambiente sano y equilibrado es también un derecho fundamental.

\section{Los enfoques de la adaptación en la Ley 30754}

Tal como lo señala el Ministerio del Ambiente (en adelante, "MINAM") en la Tercera Comunicación del Perú a la CMNUC (MINAM, 2016), el punto de partida para un proceso de adaptación es definir a la población que presenta mayores niveles de vulnerabilidad. Ahora bien, esta vulnerabilidad no debe ser considerada solo ante efectos del cambio climático, por razones geográficas, sino también por temas sociales y económicos.

En ese sentido, la Ley Marco de Cambio Climático presenta los enfoques que deben ser tomados en cuenta para la gestión integral del cambio climático. Dichos enfoques se relacionan con los criterios de vulnerabilidad, pero también con aspectos de la realidad en la cual se aplicará la norma. El siguiente cuadro presenta algunos de los once enfoques que presenta la norma (ver cuadro adjunto).

Como se desprende de la lectura de algunos de los enfoques propuestos, la Ley Marco de Cambio Climático parte de un reconocimiento de derechos fundamentales relacionados con el cambio climático, derechos que serán la columna y guía de la política en materia de cambio climático y, por ende, de la adaptación al mismo.

\section{La gestión integral del cambio climático: la adaptación es una gestión de todos}

En este acápite desarrollaremos un poco más la idea de la adaptación como una acción que comprende no solo a los diferentes niveles de gobiernos (nacional, regional y local), sino también a actores privados o no estatales. Ello, más aun cuando, como país -a nivel de ciudades- nos enfrentamos a un desarrollo sin planificación, en el cual el crecimiento poblacional se da sin un ordenamiento territorial previo, lo cual permite asentamientos alrededor de zonas de riesgo (Lanegra Quispe, 2017).

Como lo hemos señalado líneas arriba, la Ley Marco de Cambio Climático es coherente y concordante con el Acuerdo de París en señalar que la adaptación involucra a todos los niveles de gobierno de los países miembros. Así, la norma interna indica que las políticas, estrategias, planes y programas, y los proyectos de inversión deberán tener un enfoque de adaptación. Ello, bajo la participación de los actores no estatales, con especial interés en los pueblos indígenas. Estos últimos tienen un principal aporte, no solo por la diversidad cultural que presentamos como país, sino también porque la interculturalidad implica una visión diferente de desarrollo; la cual debe ser considerada desde el diálogo intercultural.

Ahora bien, la norma reconoce como autoridad nacional en materia climática al MINAM -ente rector de la política ambiental en el Perú- pero que deberá coordinar y articular con los demás sectores y niveles de gobierno. Esto es, la transversalidad del enfoque climático, lo cual podrá ser

\begin{tabular}{|c|c|}
\hline Enfoque & Comentario \\
\hline CONOCIMIENTOS TRADICIONES & $\begin{array}{l}\text { Reconoce la importancia de recuperación y revaloración de los conocimientos de pueblos } \\
\text { indígenas u originarios, por su relación con el medioambiente. }\end{array}$ \\
\hline INTERCULTURAL & $\begin{array}{l}\text { Busca el diálogo intercultural, a fin de incorporar en los planes de adaptación, la visión de } \\
\text { desarrollo y necesidades propias de los pueblos indígenas u originarios. }\end{array}$ \\
\hline IGUALDAD & $\begin{array}{l}\text { Considera el concepto de daño diferenciado. Es un principio que entra en debate ante el } \\
\text { avance en materia de género. Busca que las medidas y políticas en materia de adaptación } \\
\text { tengan un enfoque de género, en tanto plantea la igualdad entre hombres y mujeres. } \\
\text { Sin embargo, es preciso indicar que este principio debe leerse sistémicamente con el de } \\
\text { equidad. }\end{array}$ \\
\hline CUENCAS HIDROGRÁFICAS & $\begin{array}{l}\text { Orientado a la protección de cuencas, por su carácter de vulnerabilidad ante los efectos del } \\
\text { cambio climático. Principio que permite asegurar el derecho fundamental al agua. }\end{array}$ \\
\hline INTERGENERACIONAL & $\begin{array}{l}\text { Este enfoque reconoce que el daño se ha generado y que lo que nos queda es mitigar, con- } \\
\text { servar, pero sobre todo adaptarnos con miras a la preservación de generaciones futuras. } \\
\text { La lectura del mismo es un reconocimiento además a la responsabilidad con las futuras } \\
\text { generaciones, por las causas de las decisiones tomadas para la orientación de la economía } \\
\text { y el desarrollo. }\end{array}$ \\
\hline
\end{tabular}


realidad sólo si se cumple con un paso a la par, la institucionalidad ambiental. En esa línea, la norma crea la Comisión de Alto Nivel de Cambio Climático, la cual tendrá como principal objetivo la proposición de medidas de adaptación y mitigación al cambio climático y, las iNDC. Dicha Comisión será de tipo permanente y deberá considerar también las propuestas -desde la participación- de los actores no estatales. Un dato importante es que esa participación, para el caso de los pueblos indígenas, se dicta en el marco de la Ley 29785, Ley de Consulta Previa.

En relación con las medidas a adoptar para la adaptación, la Ley Marco de Cambio Climático presenta en su artículo 15 que estas deberán garantizar territorios resilientes y sostenibles. Ello, mediante la priorización de recursos hídricos, ciudades sostenibles y prevención de riesgos; lo cual involucra la gestión de los mismos.

Hasta este punto, se denota que la Ley Marco de Cambio Climático entiende a la adaptación como una obligación y una medida no solo de hacer frente a los impactos ya generados, sino también para preservar el medioambiente, desde diferentes enfoques de derechos.

La idea señalada en el párrafo precedente puede lograrse, entre otros factores, si cambiamos el paradigma de inversión que impera en nuestras instituciones. Lograr la adaptación implica que la inversión deberá ser responsable y en armonía con el medioambiente y el reconocimiento de derechos colectivos de pueblos indígenas. Por ello, la Ley Marco de Cambio Climático consideró en su Primera Disposición Complementaria Final que los proyectos de inversión pública y privada deberán contribuir con las medidas de adaptación y con las iNDC. Así, una vez más, reconocemos que hacer frente a los impactos es una obligación de todos, pero también lo es reconocer la responsabilidad en dichos impactos.

Adicionalmente, con relación al actor privado, la norma interna considera en su Segunda Disposición Final Transitoria que los proyectos de inversión en el marco del Sistema Nacional de Evaluación de Impacto Ambiental deberán considerar en sus instrumentos de gestión ambiental la identificación de medidas de adaptación al cambio climático. Como era de esperarse, este punto generó un fuerte debate y resistencia, el cual no encontró asidero, en tanto ello está reconocido en el propio Acuerdo de París.
Finalmente, debemos señalar que la importancia de la Ley Marco de Cambio Climático en materia de adaptación climática es el cambio del paradigma ante un modelo económico que permita la gestión integral, la sostenibilidad y, por encima de todo, el reconocimiento del derecho fundamental al medioambiente.

\section{CONCLUSIONES}

El cambio climático implica una revisión de nuestras políticas ambientales y de la institucionalidad de las mismas. Los impactos de dicho cambio han obligado a diferentes países a asumir compromisos claros para hacer frente a ellos y, por otro lado, a modo de reconocimiento de la responsabilidad producto de las actividades humanas. El cambio climático, más que un curso natural, es producto de un inadecuado manejo de la actividad humana, especialmente aquella relacionada a la economía y la inversión.

Esto último nos permite señalar que la economía llegó a la atmósfera. El modelo de desarrollo asumido, en el cual sostenemos nuestra economía en la extracción y utilización de recursos naturales, no ha permitido ni preservar ni conservar -ni mucho menos garantizar- la sostenibilidad ambiental. Ante ello, aun cuando suene fatalista, el camino es la adaptación.

En ese camino, el Acuerdo de París viene cumpliendo un rol importante para la pausa necesaria a pensar en el qué estamos haciendo. El Perú no se quedó atrás en ese quehacer y planteó la adaptación no solo -de manera referencial- en las iNDC, sino también mediante una Ley Marco de Cambio Climático que permite -entre otros- orientar de manera adecuada la aplicación y diseño de la política de adaptación.

Sin embargo, la norma -desde la función social del derecho- no tendrá mayor impacto sin una decisión política que permita el cambio de paradigma a favor de la institucionalidad ambiental. Ese cambio que debe considerar factores interculturales y sociales.

Finalmente, la responsabilidad de hacer frente al cambio climático recae en el Estado (desde cada poder y nivel de gobierno) y en la ciudadanía. Responsabilidad para con las generaciones futuras, a quienes podremos dejar un territorio devastado o uno en el cual minimicemos los impactos y logremos el equilibrio entre naturaleza, economía y sociedad. 


\section{REFERENCIAS}

Comisión Económica para América Latina y el Caribe (CEPAL) (2012). La Economía del Cambio Climático en Centroamérica: Síntesis 2012. Ciudad de México: Naciones Unidas.

Gudynas, E. (2004). Ecología, Economía y Ética del Desarrollo Sostenible. Montevideo: CLAES.

Equipo de redacción de BBC Mundo. (2005). Las claves del cambio climático. BBC Mundo 10 de febrero. Recuperado de: http://news. bbc.co.uk/hi/spanish/specials/2005/kioto/ newsid_4232000/4232573.stm.

Iguíñiz Echeverría, J. (1996). Definiciones de desarrollo y experiencias de género. Lima: Instituto Bartolomé de las Casas.

Lanegra Quispe, I. (2017). ¿Qué es el cambio climático? Calentamiento Global y Sociedad. Lima: Editorial Planeta.

(2008). El (ausente) Estado Ambiental. Razones para la reforma de las instituciones y las organizaciones públicas ambientales en el Perú. Lima: CDE.

Magrin, G. O. (2015). Adaptación al cambio climático en América Latina y el Caribe. Santiago de Chile: Naciones Unidas.

Mendoza, M. (2009). Justicia Climática: Una tarea pendiente. Managua: Centro de Estudios Internacionales.

Ministerio del Ambiente (2016). El Perú y Cambio Climático: Tercera Comunicación Nacional del Perú a la Convención Marco de las $\mathrm{Na}$ ciones Unidos sobre Cambio Climático. Lima. Recuperado de: http://www.minam.gob. pe/wp-content/uploads/2016/05/TerceraComunicaci\%C3\%B3n.pdf.

Mulugetta, Y.; Hagan, E. B. y D. Kammen (2019). Energy access for sustainable development. DOI: 10.1088/1748-9326/aaf449
Stratus Consulting Inc., Universidad Nacional Autónoma de México y Colegio de Sonora. (2004). Adaptación al Cambio Climático: Hermosillo, Sonora, un caso de estudio. Ciudad de México: Instituto Nacional de Ecología, Secretaría de Medio Ambiente y Recursos Naturales y United States Environmental Protection Agency.

Watt, L. (2016). Politics of Anthropocene Consumption: Dipesh Chakrabarty and Three College Courses. En: RCC Perspectives: Transformations in Environment and Society 2, pp. 73-79. DOI:10.5282/rcc/7448

Zwarteveen, M. (2013). El Cambio Climático desde la Perspectiva de la Justicia Social. En: Hoogesteger, J. y P. Urteaga (eds.). Agua e Inequidad. Discursos, políticas y medios de vida en la región andina. Lima: Instituto de Estudios Peruanos.

\section{LEGISLACIÓN, JURISPRUDENCIA Y OTROS DOCU- MENTOS LEGALES}

Congreso de la República del Perú (2018). Diario de Debates. Segunda legislatura ordinaria de 2017. Recuperado de: http://www2.congreso. gob.pe/Sicr/DiarioDebates/Publicad.nsf/Sesio nesPleno/05256D6E0073DFE9052582520059 5BF5/\$FILE/SLO-2017-2.pdf.

Convención Marco de las Naciones Unidas sobre el Cambio Climático (UNFCCC) (2015). Aprobación del Acuerdo de Paris. Recuperado de: https://unfccc.int/files/meetings/paris_ nov_2015/application/pdf/paris_agreement_ spanish_.pdf.

(1998). Aprobación de Kyoto de la Convención Marco de las Naciones Unidas sobre Cambio Climático. Recuperado de: https://unfccc.int/ resource/docs/convkp/kpspan.pdf.

Ley 30754. Ley Marco sobre Cambio Climático. En: Diario El Peruano, 18 de abril de 2018. 